\title{
Lithium concentration dependent structure and mechanics of amorphous silicon
}

Cite as: J. Appl. Phys. 119, 245103 (2016); https://doi.org/10.1063/1.4954683

Submitted: 21 March 2016 . Accepted: 11 June 2016 . Published Online: 23 June 2016

H. S. Sitinamaluwa, M. C. Wang, G. Will, W. Senadeera, S. Zhang, and C. Yan

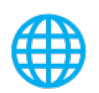

View Online

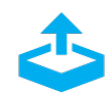

Export Citation

\section{ARTICLES YOU MAY BE INTERESTED IN}

Lithium diffusion in silicon and induced structure disorder: A molecular dynamics study

AIP Advances 3, 112102 (2013); https://doi.org/10.1063/1.4829440

Rate-dependent stress evolution in nanostructured Si anodes upon lithiation Applied Physics Letters 109, 163903 (2016); https://doi.org/10.1063/1.4964515

Theoretical prediction of fracture conditions for delithiation in silicon anode of lithium ion battery

APL Materials 5, 106101 (2017); https://doi.org/10.1063/1.4997978

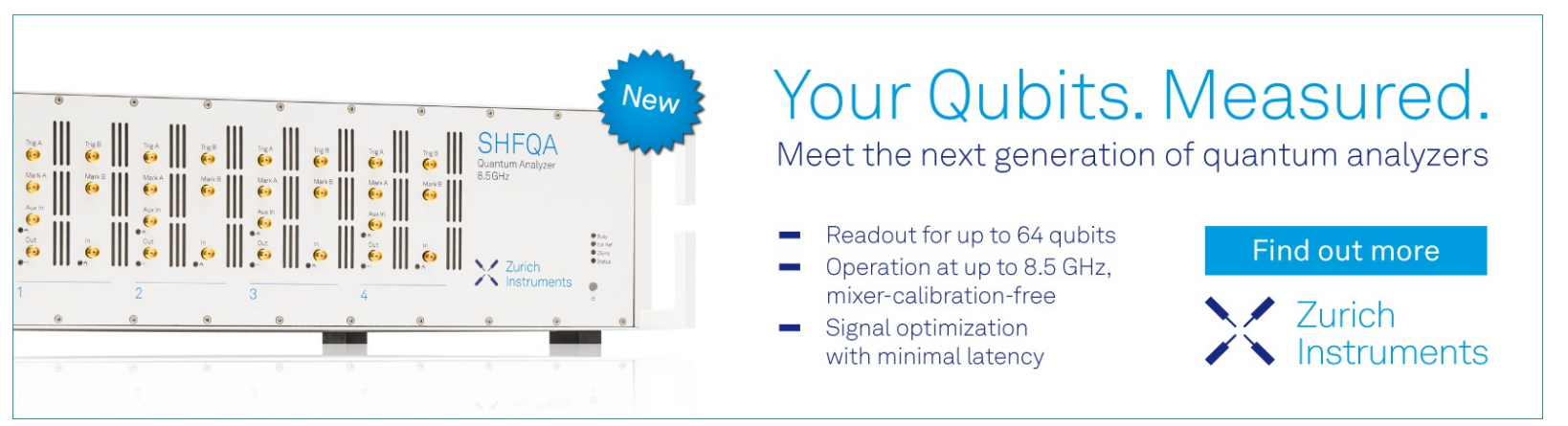




\title{
Lithium concentration dependent structure and mechanics of amorphous silicon
}

\author{
H. S. Sitinamaluwa, ${ }^{1}$ M. C. Wang, ${ }^{1}$ G. Will, ${ }^{1}$ W. Senadeera, ${ }^{1}$ S. Zhang,${ }^{2}$ and C. Yan ${ }^{1, a)}$ \\ ${ }^{1}$ School of Chemistry, Physics and Mechanical Engineering, Queensland University of Technology (QUT), \\ Brisbane QLD 4001, Australia \\ ${ }^{2}$ Centre for Clean Environment and Energy, Environmental Futures Research Institute and Griffith School \\ of Environment, Gold Coast Campus, Griffith University, QLD 4222, Australia
}

(Received 21 March 2016; accepted 11 June 2016; published online 23 June 2016)

\begin{abstract}
A better understanding of lithium-silicon alloying mechanisms and associated mechanical behavior is essential for the design of Si-based electrodes for Li-ion batteries. Unfortunately, the relationship between the dynamic mechanical response and microstructure evolution during lithiation and delithiation has not been well understood. We use molecular dynamic simulations to investigate lithiated amorphous silicon with a focus to the evolution of its microstructure, phase composition, and stress generation. The results show that the formation of $\mathrm{Li}_{x} \mathrm{Si}$ alloy phase is via different mechanisms, depending on Li concentration. In these alloy phases, the increase in Li concentration results in reduction of modulus of elasticity and fracture strength but increase in ductility in tension. For a $\mathrm{Li}_{x} \mathrm{Si}$ system with uniform $\mathrm{Li}$ distribution, volume change induced stress is well below the fracture strength in tension. Published by AIP Publishing. [http://dx.doi.org/10.1063/1.4954683]
\end{abstract}

\section{INTRODUCTION}

Li-ion battery (LIB) is increasingly used in various portable electronic devices and electric vehicles (EV) due to high energy/power density and long lifespan. ${ }^{1,2}$ To reduce the structural weight in transportation systems, novel Li-ion batteries with higher energy density is required. ${ }^{3}$ Silicon, with its highest known theoretical specific capacity (i.e., $4200 \mathrm{~mA} \mathrm{~h} \mathrm{~g}^{-1}$ ) has emerged as a promising candidate as $\mathrm{Li}-$ ion battery anode material. ${ }^{4}$ However, mechanical integrity of Si remains as a challenge due to its huge volume expansion $(\sim 300 \%)$. ${ }^{5}$ When subjected to constraints imposed by electrode architecture, such enormous expansion can cause mechanical stress and fracture. ${ }^{5,6}$ Various strategies have been developed to improve the mechanical stability of silicon, including nanostructured electrodes, ${ }^{7,8}$ flexible binder materials, ${ }^{9}$ and carbon based hybrids. ${ }^{10}$ Recently, there has been increasing interest in amorphous silicon (a-Si), owing to its robust lithiation behavior compared with its crystalline counterpart. ${ }^{11,12}$

Lithiation of amorphous silicon occurs via formation of metastable $\mathrm{Li}_{x} \mathrm{Si}$ alloy phases ${ }_{1}^{13}$ identified as $\mathrm{Li}_{15} \mathrm{Si}_{4}$ for fully lithiated silicon. ${ }^{14}$ McDowell et al. showed that a-Si nanospheres undergo up to $332 \%$ volume expansion at full lithiation. ${ }^{11}$ Several authors have examined mechanical behavior of electrochemically lithiated silicon. Berla et al. ${ }^{15}$ performed nanoindentation tests to evaluate the modulus and hardness of lithiated amorphous silicon as a function of $\mathrm{Li}$ concentration in active material. Also, in-situ atomic force microscopy (AFM) and transmission electron microscopy (TEM) have been used to measure Young's modulus and tensile strength of lithiated silicon. ${ }^{16,17}$ Furthermore, Berla et al. showed viscoelastic flow and creep behavior in lithiated silicon, indicating the possibility of plastic deformation

a)E-mail: c2.yan@qut.edu.au under high Li concentrations. ${ }^{15}$ In addition, recent experiments and theoretical studies suggest that lithiation induced plasticity can effectively prevent fracture. ${ }^{18,19}$ These studies demonstrated a strong dependence of mechanical properties on phase composition. From recent first-principle calculations, the microstructural evolution such as $\mathrm{Si}-\mathrm{Si}$ bond breaking and regeneration has been explained as responsible for the composition-dependent mechanical properties of lithiated silicon. $^{20-22}$ However, due to limitations in length-scale, the model cells in those first-principles calculations cannot fully represent the typical a-Si. Therefore, more work is required to understand the underlying structure-property relationship in lithiated silicon.

In this study, we use molecular dynamic (MD) simulations to investigate the volume expansion and stress development of amorphous silicon during lithiation and their dependence with phase composition and atomic structures. Through quantitative analysis of atomic structures, we find that the formation of $\mathrm{Li}_{x} \mathrm{Si}$ phase is via different mechanisms, depending on the Li concentration. Furthermore, our results indicate that the changed atomic mobility in lithiated silicon structures play a major role in transient mechanical properties. The outcomes of this work are believed to be useful for design of Si based electrodes with improved mechanical stability.

\section{METHODS}

In the molecular dynamic (MD) simulation, the initial amorphous silicon (a-Si) atomic model is a cubic unit with a side length $2.7 \mathrm{~nm}$, which contains 1000 silicon atoms as shown in Figure 1(a). The a-Si model was created by quenching molten silicon from $2400^{\circ} \mathrm{C}$ to room temperature at a cooling rate of $10^{12} \mathrm{~K} \mathrm{~s}^{-1}$. A modified Stillinger-Weber potential for a-Si was used. ${ }^{23}$ After structural relaxation, the density of the generated a-Si was calculated as $2.291 \mathrm{~g} \mathrm{~cm}^{-3}$. The a-Si 

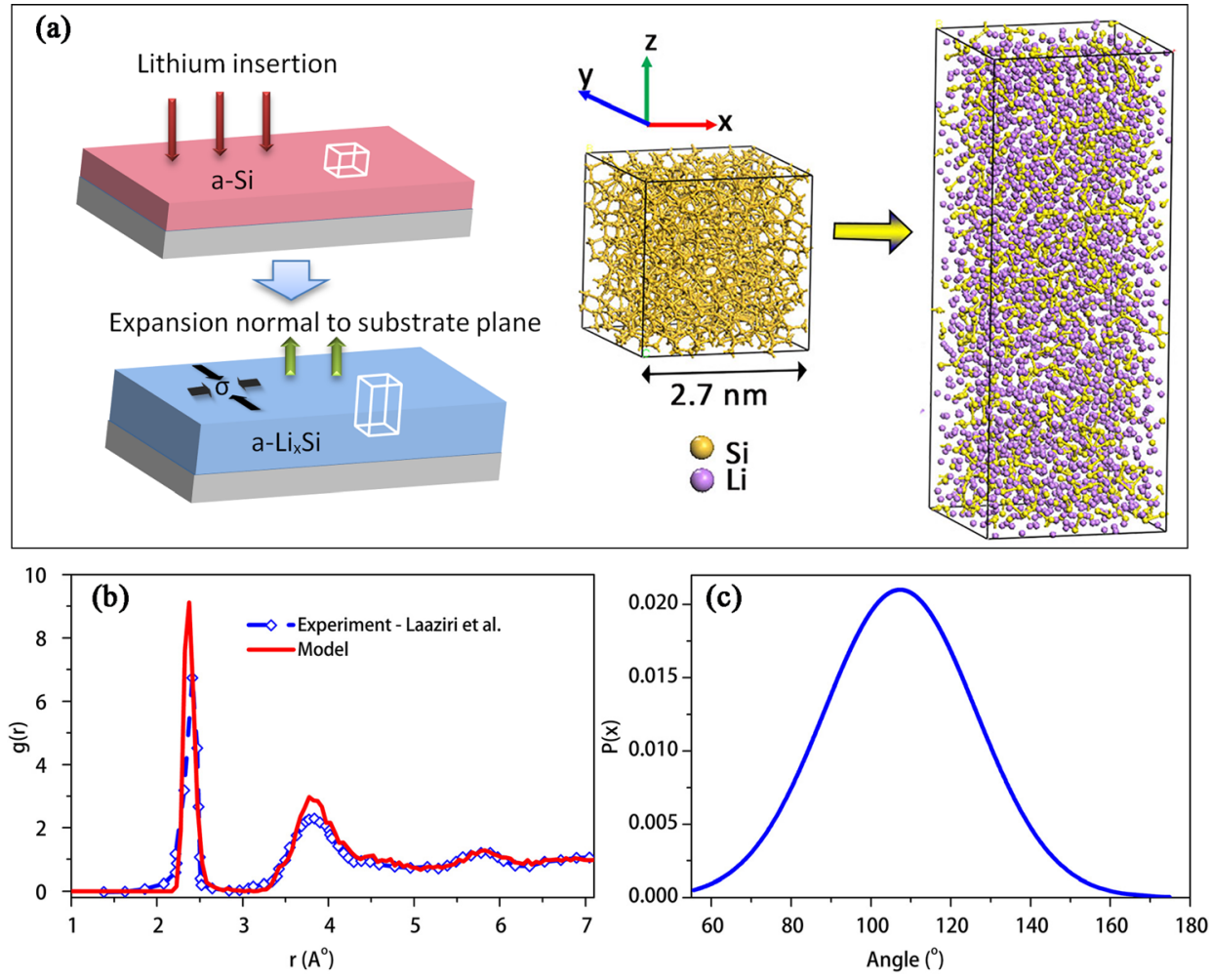

FIG. 1. (a) Schematic illustration (left panel) and atomic structure (right panel) of the lithiation process of the 1000-atom (a-Si) model constrained by a substrate. (b) A comparison between the radial distribution function (RDF) results determined by MD simulation and previous experiment. ${ }^{24}$ (c) Bond angle distribution function of the a-Si atomic model. atomic model was validated by comparing the radial distribution function (RDF) with the existing experimental data, ${ }^{24}$ as shown in Figure 1(b). The broad first-neighbor peak and the absence of well-defined higher-neighbor peaks in the RDF confirm its amorphous structure. The average Si-Si coordination number $(\mathrm{CN})$ was estimated to be about 4 , confirming a tetrahedral structure as reported in literature. ${ }^{24}$ Further, as shown in Figure 1(c), the average bond angle is $107.3^{\circ}$ with standard deviation $18.9^{\circ}$, in good agreement with previous simulations. $^{25,26}$

For crystalline silicon, lithiation may occur via a twophase mechanism; ${ }^{27,28}$ however, detailed lithiation mechanism of a-Si still remains unclear. ${ }^{29-32}$ To understand the effect of $\mathrm{Li}$ concentration, it is reasonable to assume a uniform dispersion of $\mathrm{Li}$ ion in the a-Si. To simulate lithiation-delithiation, a stepwise Li insertion and extraction procedure was used. ${ }^{33}$ For each insertion step, $25 \mathrm{Li}$ atoms were randomly distributed in the a-Si cell, and the structural relaxation was carried out. Energy minimization was achieved when the forces on atoms were less than $10^{-8} \mathrm{eV} / \AA$. The simulation cell was equilibrated at $300 \mathrm{~K}$ in isothermal-isobaric (NPT) ensemble for further 10 ps. This procedure was repeated until the desired $\mathrm{Li}$ concentration was obtained. Similarly, delithiation was also simulated stepwise in which $25 \mathrm{Li}$ atoms were removed randomly, and the structure was relaxed in the NPT ensemble. Atomic interactions during $\mathrm{Li}$ insertion and extraction were defined by using modified embedded atom method (MEAM) based interatomic potential for Li-Si alloys. ${ }^{34}$

When the a-Si anode is attached to a rigid substrate as shown in Figure 1(a), non-zero intrinsic stress generates along the substrate (in-plane) direction due to volume expansion. ${ }^{19,33,35}$ To estimate such intrinsic stress in lithiated a-Si, the in-plane ( $x$ - and $y$-axis) lengths of the simulation cell were fixed to mimic the constraint induced by the rigid substrate, and the volume expansion along the thickness ( $z$ axis) direction was set free. Based on the virial theorem, ${ }^{36,37}$ the atomic stresses along the in-plane direction were calculated after each lithiation step by running MD simulations under the NVT ensemble for further $10 \mathrm{ps}$. Li atoms distribution in the cell is approximately uniform, to avoid the concentration gradient dependent stress. ${ }^{38}$ To understand the mechanical properties of lithiated a-Si, uniaxial tensile tests along $x$ axis (as shown in Figure 1(a)) were also conducted to calculate the fracture strengths of several lithiated a- $\mathrm{Li}_{x} \mathrm{Si}$ structures at a constant strain rate of $10^{8} \mathrm{~s}^{-1} \cdot{ }^{39,40}$ All simulations were performed using LAMMPS software, ${ }^{41}$ which has been used for simulating mechanical properties of nanomaterials in our previous studies. $^{42-44}$ Atomic structure analysis and visualization were carried out using OVITO ${ }^{45}$ and $\mathrm{VMD}^{46}$ postprocessing tools.

\section{RESULTS AND DISCUSSION}

\section{A. Lithiation-induced microstructural transformations}

Amorphous silicon has a disordered tetrahedral structure where Si atoms are covalently bonded forming continuous random network $(\mathrm{CRN}) .{ }^{24}$ With lithiation process, $\mathrm{Li}$ atoms react with $\mathrm{Si}$ to form $\mathrm{Li}_{x} \mathrm{Si}$ alloy phase, with $\mathrm{Li}$ to $\mathrm{Si}$ ratio $(x)$ increasing up to $4.4{ }^{4}$ In this work, $\mathrm{Li}_{x} \mathrm{Si}$ alloys $(x=0$ to 3.75$)$ were simulated as these $\mathrm{Li}$ to $\mathrm{Si}$ ratios have been observed in room temperature lithiation. ${ }^{14}$ Upon lithiation, gradual breakdown of a-Si CRN and formation of new Li-Si bonds are observed, leading to volume expansion. The maximum volume expansion observed in current model at full lithiation $\left(\mathrm{Li}_{3.75} \mathrm{Si}\right)$ was $283 \%$, in agreement with experiment. ${ }^{11}$ 


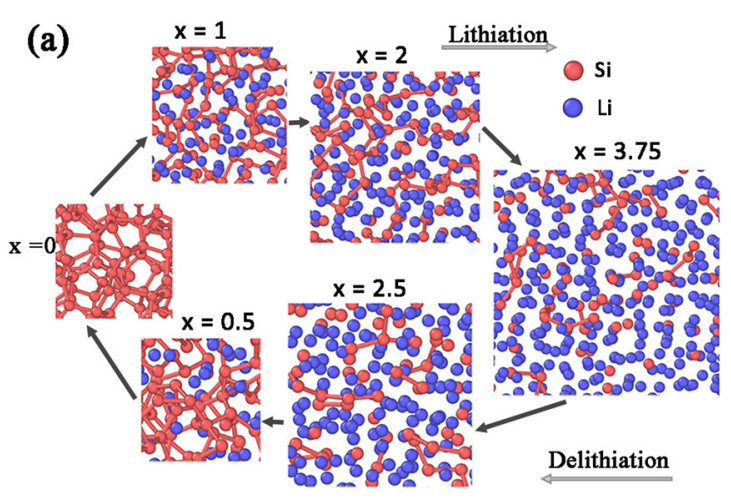

Figure 2(a) shows the atomic structures at different stages of lithiation. For clarification, only $\mathrm{Si}-\mathrm{Si}$ bonds are shown. The radial distribution function (RDF) of Si-Si and Li-Si pairs at different stages of lithiation was evaluated. RDF is an indicator of how atoms are packed in space and can provide short and medium range structure information, which can be expressed as

$$
g(r)=\frac{N(r, r+\Delta r)}{4 \pi r^{2} \Delta r \rho},
$$

where $r$ is the distance between the reference particle and found particle; $N(r, r+\Delta r)$ represents the number of atoms in specified pairs within the region $(r, r+\Delta r)$, and $\rho$ is the total bulk density of the considered particle.

Figure 2(b) shows the Si-Si RDFs for a-Si and a- $\mathrm{Li}_{x} \mathrm{Si}$ at different composition. The initial bulk silicon structure $(x=0)$ do not show sharp second neighbor peak due to the lack of long range ordered atomic structure. The largest $\mathrm{Si}$ Si pair distance corresponding to the nearest neighbor peak is $2.6 \AA$, which is the cut-off distance for the $\mathrm{Si}-\mathrm{Si}$ bond. Similarly, the Si-Li bond cut-off distance is calculated as 3.3 $\mathrm{A}$. The $\mathrm{Si}-\mathrm{Si}$ RDFs as a function of $\mathrm{Li}$ concentration, during lithiation and delithiation, are shown in Figure 2(b). With the increase in $\mathrm{Li}$, the $\mathrm{Si}-\mathrm{Si}$ nearest neighbor peak shifts to larger pair distance, indicating weakened $\mathrm{Si}-\mathrm{Si}$ bonds. ${ }^{47}$ For example, at $x=0$, the largest $\mathrm{Si}-\mathrm{Si}$ pair distance is $2.6 \AA$, and increases to $2.9 \AA$ at full lithiation. Also, the reduced peak height during lithiation indicates the break of Si-Si bonds. During delithiation, the Si-Si bonds can be recovered, as indicated by the increased peak height. Further, the $\mathrm{Si}-\mathrm{Si}$ nearest neighbor peak is visible at $x=3.75$, associated with the unbroken $\mathrm{Si}-\mathrm{Si}$ bonds. The bond break and recovery can be observed in the atomic structures shown in Figure 2(a).

The atomic coordination numbers $(\mathrm{CN})$ were calculated by taking integral of the nearest neighbor peak of RDF for corresponding atomic pair. In Figure 3(a), Si-Si CN represents the average number of coordinated $\mathrm{Si}$, while $\mathrm{Si}-\mathrm{Li} \mathrm{CN}$ represents the average number of $\mathrm{Li}$ coordinated by a $\mathrm{Si}$ atom. At $x=0, \mathrm{CN}$ of a-Si structure is $\sim 4$, as atoms are tetrahedrally bonded. With increasing Li concentration, Si-Si CN decreases due to break of Si-Si bonds. Simultaneously, Si-Li $\mathrm{CN}$ increases with $\mathrm{Li}$ invasion due to the formation of new $\mathrm{Si}$-Li bonds. The CN curves show different trends when Li concentration $(x)$ is above and below 2.5. Below $x=2.5$, $\mathrm{Si}-\mathrm{Si} \mathrm{CN}$ gradually decreases and is saturated around $\sim 1$. This is accompanied by the increase of $\mathrm{Si}-\mathrm{Li} \mathrm{CN}$, indicating that a rapid breakdown of $\mathrm{Si}-\mathrm{Si}$ bonds takes place below $x=2.5$. Also, $\mathrm{Si}-\mathrm{Si} \mathrm{CN}$ is retained at $\sim 4$ until $x$ reaches 0.25 . First-principles calculation indicates that $\mathrm{Li}$ atoms initially take the positions at interstices of larger silicon rings, ${ }^{20}$ with only minor distortions to the a-Si continuous random network $(\mathrm{CRN})$. It is also reported that four $\mathrm{Li}$ atoms at the tetrahedral sites can result in a very small energy barrier $(0.08 \mathrm{eV})$ against the break of $\mathrm{Si}-\mathrm{Si}$ bond, and thus, it is believed that at least four $\mathrm{Li}$ atoms are needed to weaken and break a Si-Si bond. ${ }^{22}$

We further analyze the breakdown of amorphous silicon structure via cluster analysis. A group of silicon atoms (two or more) connected with the $\mathrm{Si}-\mathrm{Si}$ bonds is considered as a silicon cluster. At $x=0$, the bulk silicon structure is one big cluster of 1000 atoms, and every silicon atom is a part of tetrahedral CRN. It is interesting to note that the silicon structure does not start to break until $x$ exceeds $\sim 0.25$. At $x=3.75$, silicon atoms are mainly arranged in three different
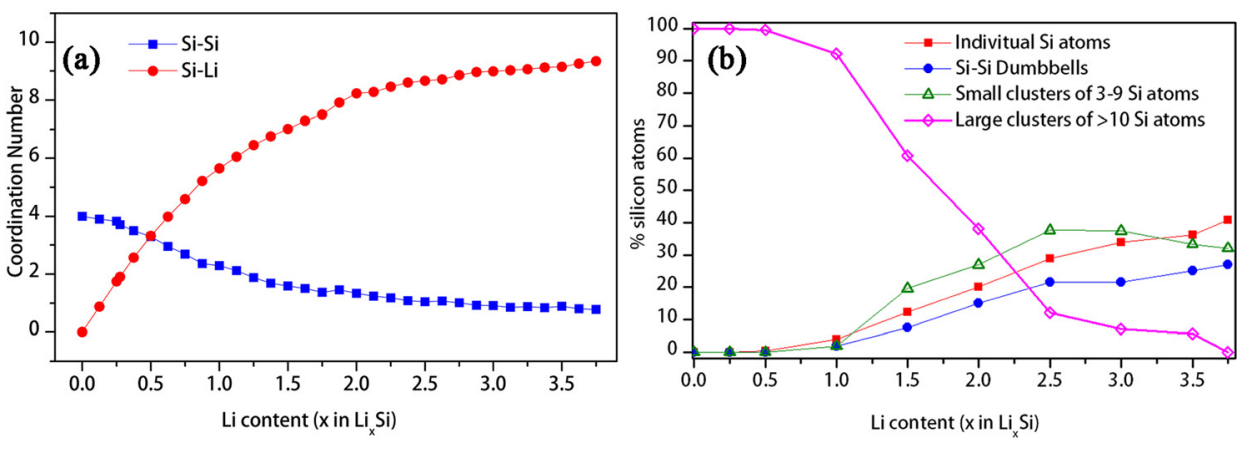

FIG. 3. (a) Si-Si and Si-Li coordination numbers during lithiation as a function of $\mathrm{Li}$ concentration. (b) Breakdown of silicon CRN into number of smaller structures during lithiation via cluster analysis. 


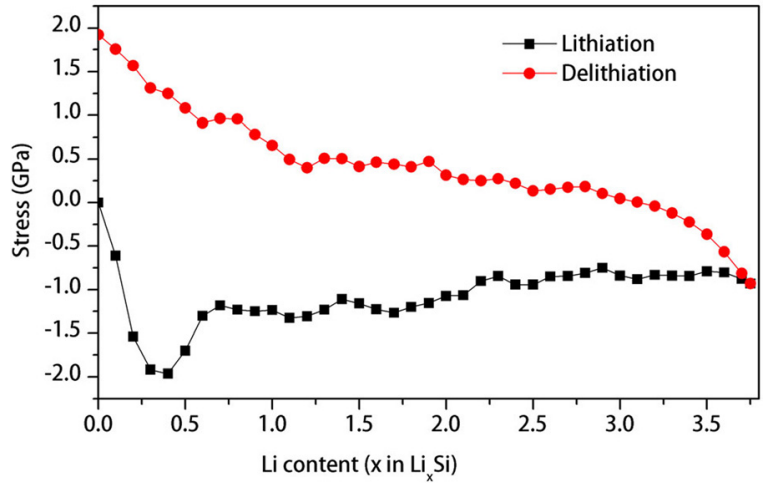

FIG. 4. Stress development in constrained a-Si thin film during lithiation/ delithiation process.

structures: (i) lithiated individual silicon atoms, (ii) lithiated Si-Si dumbbells, and (iii) lithiated silicon clusters of 3-9 atoms. Figure 3(b) gives the percentage of these structures as a function of Li concentration. In Figure 3(b), a rapid breakdown of large silicon clusters and formation of smaller structures observed up to $x \sim 2.5$. At $x=2.5$, about $90 \%$ silicon atoms are in the form of dumbbells, small clusters or individual atoms and the rest $10 \%$ are unbroken larger clusters. This suggests that $\sim 90 \% \mathrm{Si}$ structure's break down takes place below $x=2.5$. As indicated in Figure 3(a), a silicon atom has an average of $8.3 \mathrm{Li}$ neighbors at $x=2.5$, which increases up to 9.3 in full lithiation $(x=3.75)$. Therefore, lithiation beyond $x=2.5$ creates more Li-rich phases around silicon clusters without significant structural change.

Based on the structure analysis, lithiation is featured by a three-step process. The first step occurs below $x \sim 0.25$, where $\mathrm{Li}$ atoms occupy interstitial sites in silicon structure with only minor distortions to a-Si CRN. The second step corresponds to the region between $x \sim 0.25$ and $x \sim 2.5$, where a-Si CRN structure undergoes considerable transformations via a rapid $\mathrm{Si}-\mathrm{Si}$ bond breaking. In this step, the a-Si structure is mainly broken down to lithiated silicon atoms, dumbbells, and smaller clusters. Above $x \sim 2.5$, further lithiation creates more Li-rich phases with relatively less $\mathrm{Si}-\mathrm{Si}$ bond breaking.

\section{B. Effects of structural transformation on stress and deformation of a-Si electrode}

Figure 4 shows lithiation-delithiation induced stress in a thin film electrode as a function of $\mathrm{Li}$ concentration.
During lithiation, a sharp increase in compressive stress up to $\sim 2 \mathrm{GPa}$ is observed as $x=0.3$. It is followed by stress relaxation, where compressive stress decreases to $\sim 1 \mathrm{GPa}$ as $x=2.5$. Finally, almost constant stress of $\sim 1 \mathrm{GPa}$ is observed for the rest of lithiation. It is interesting to note that lithiation induced stress shows a clear three-step response which corresponds to the structural transformations described in Section III A.

In the first step, Li atoms occupy interstices between silicon rings, without breaking the tetrahedral CRN. As the covalent $\mathrm{Si}-\mathrm{Si}$ bonds dominating the structure at this stage, volume expansion creates significant compressive stress. As Li concentration increases up to $x=2.5$, silicon structure breaks down to form $\mathrm{Si}-\mathrm{Li}$ phases, with apparent stress relaxation. Zhao et al. ${ }^{22}$ demonstrated that Si-Li pair has a mixed ionic-covalent bond, weaker than covalent $\mathrm{Si}-\mathrm{Si}$ bonds. Thus, stress relaxation observed at this stage may be attributed to the increased number of weak Li-Si pairs. This behavior is also described as plastic flow of $\mathrm{Li}_{x} \mathrm{Si}$ structure. ${ }^{19,22}$ With more Li invasion (up to $x \sim 2.5$ ), compressive stress gradually decreases to $\sim 1 \mathrm{GPa}$. Interestingly, when $x>2.5$, no further increase in stress is observed. In general, plastic flow at a constant stress is usually observed when diffusing atoms are trapped in structural defects. For example, high temperature creep of some alloys is found to occur under constant stress, due to trapping of diffusing atoms in grain boundaries. ${ }^{48}$ In this work, silicon clusters may act as diffusion barrier, contributing to constant-stress flow. During delithiation, the model volume starts to contract and elastic unloading occurs, where restriction from the substrate converts the stress from compression to tension, as shown in Figure 4. There is a sharp increase in tensile stress at lower $\mathrm{Li}$ concentrations, i.e., below $\sim 0.5$. This is associated with the possible recovery of covalent $\mathrm{Si}$ bonds at low Li-ion concentrations, which results in significant increases in stress along with volume contraction.

To understand the intrinsic mechanical behavior of the lithiated $\mathrm{Si}$, a uniaxial tensile test was simulated in several $\mathrm{Li}_{x} \mathrm{Si}$ structures. The stress-strain curves of the uniaxial tensile tests are shown in Figure 5(a). As shown in Table I, the modulus of elasticity of the lithiated silicon decreases with increasing $\mathrm{Li}$ concentration. The stress-strain response of those $\mathrm{Li}_{x} \mathrm{Si}$ with $x=0$ and $x=0.25$ is like brittle materials, and no notable plastic deformation is observed. In contrast, considerable plastic deformation can be observed in the $\mathrm{Li}_{x} \mathrm{Si}$ of high $\mathrm{Li}$ concentrations, which can be attributed to
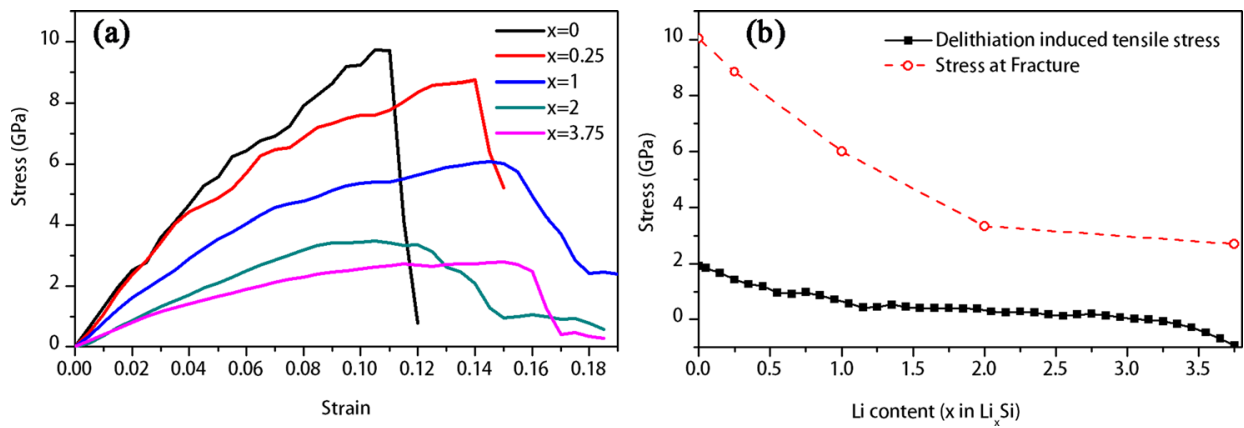

FIG. 5. (a) Stress-strain curves of a-Si and $\mathrm{a}-\mathrm{Li}_{x} \mathrm{Si}$ structure in uniaxial tension. (b) A comparison of tensile fracture stresses of $\mathrm{Li}_{x} \mathrm{Si}$ structures with delithiation induced tensile stress. 
TABLE I. Mechanical characteristics of $\mathrm{Li}_{x} \mathrm{Si}$ alloys and mean square displacement of $\mathrm{Si}$ atoms $(\mathrm{MSD})_{\mathrm{Si}}$ during tensile test.

\begin{tabular}{lccc}
\hline \hline Structure & $\begin{array}{c}\text { Modulus of } \\
\text { elasticity (GPa) }\end{array}$ & $\begin{array}{c}\text { Stress at } \\
\text { fracture }(\mathrm{GPa})\end{array}$ & $\begin{array}{r}(\mathrm{MSD})_{\mathrm{Si}} \text { after } \\
1000 \mathrm{ps}(\AA)^{2}\end{array}$ \\
\hline Bulk a-Si & 125.6 & 10.0 & 12.6 \\
$\mathrm{Li}_{0.25} \mathrm{Si}$ & 118.5 & 8.8 & 13.8 \\
$\mathrm{LiSi}_{\mathrm{Li}}$ & 80.7 & 6.0 & 27.8 \\
$\mathrm{Li}_{2} \mathrm{Si}$ & 43.6 & 3.3 & 62.3 \\
$\mathrm{Li}_{3.75} \mathrm{Si}$ & 39.8 & 2.7 & 198.8 \\
\hline \hline
\end{tabular}

the Li-rich phases with higher mobility. To further confirm this, the total mean square displacement (MSD) of silicon atoms during the tensile test was evaluated, as shown in Table I. It is clear that MSD increases with increasing Li, indicating higher atomic mobility with higher number of LiSi pairs.

Figure 5(b) shows the fracture stress of the $\mathrm{Li}_{x} \mathrm{Si}$ structures in the tensile test as a function of $\mathrm{Li}$ concentration. The fracture stress is found to be decreasing with the increase in $\mathrm{Li}$ concentration. In Figure 5(b), the simulated tensile stress during delithiation is also included for comparison. It is clear that the delithiation induced stress well below the fracture stresses in tension. This implies that the tensile stress induced by volume change in the constrained model is not sufficient to trigger fracture. Therefore, amorphous silicon electrodes may fail under repeated compressive-tensile stresses during charge-discharge cycles. Further work along this direction is required.

\section{CONCLUSIONS}

In this work, molecular dynamic simulation was used to evaluate the evolution of microstructure and mechanical stresses in amorphous silicon under electrochemical lithiation-delithiation process. Through the short and medium range atomic structure analysis, it was found that $\mathrm{Li}-\mathrm{Si}$ alloying reaction occurs via a three-stage mechanism. First, Li atoms occupy interstitial sites of amorphous silicon, followed by the second stage, i.e., rapid breaking of the original $\mathrm{Si}-\mathrm{Si}$ structure to form $\sim \mathrm{Li}_{2.5} \mathrm{Si}$. Further lithiation leads to the formation of $\mathrm{Li}_{3.75} \mathrm{Si}$, accompanied by a relatively less bond breaking. At full lithiation $\left(\mathrm{Li}_{15} \mathrm{Si}_{4}\right)$, the lithiated $\mathrm{Si}-\mathrm{Si}$ dumbbells and small silicon clusters dominate the structure. With increasing Li concentration, reduced elastic modulus and fracture strength but increased ductility is observed in $\mathrm{Li}_{x} \mathrm{Si}$ phases. Cyclic compressive-tensile stresses can be generated in geometrically laterally constrained amorphous silicon during lithiation-delithiation process. Interestingly, these stresses are found to be well below the fracture stress in tension, implying that the mechanical damage in the Si-based Li battery electrodes is likely to be triggered by the cyclic tensile and compressive stresses.

\section{ACKNOWLEDGMENTS}

The authors wish to acknowledge the High Performance Computing (HPC) and CARF at QUT for access to their facilities. The work was partially supported by the ARC DP project (DP150101717). H.S.S. would like to acknowledge Queensland University of Technology for APA, IPRS, and QUT excellence top up scholarships.

${ }^{1}$ J.-M. Tarascon and M. Armand, Nature 414, 359 (2001).

${ }^{2}$ K. E. Aifantis, S. A. Hackney, and R. V. Kumar, High Energy Density Lithium Batteries: Materials, Engineering, Applications (Wiley-VCH, Weinheim, 2010).

${ }^{3}$ M. S. Whittingham, Proc. IEEE 100, 1518 (2012).

${ }^{4}$ W.-J. Zhang, J. Power Sources 196, 13 (2011).

${ }^{5}$ A. Mukhopadhyay and B. W. Sheldon, Prog. Mater. Sci. 63, 58 (2014).

${ }^{6}$ X. Gao, Z. Ma, W. Jiang, P. Zhang, Y. Wang, Y. Pan, and C. Lu, J. Power Sources 311, 21 (2016).

${ }^{7}$ J. R. Szczech and S. Jin, Energy Environ. Sci. 4, 56 (2011).

${ }^{8} \mathrm{H}$. Wu and Y. Cui, Nano Today 7, 414 (2012).

${ }^{9}$ M. Ling, Y. Xu, H. Zhao, X. Gu, J. Qiu, S. Li, M. Wu, X. Song, C. Yan, and G. Liu, Nano Energy 12, 178 (2015).

${ }^{10}$ H. Sitinamaluwa, S. Zhang, W. Senadeera, G. Will, and C. Yan, Mater. Technol. (published online).

${ }^{11}$ M. T. McDowell, S. W. Lee, J. T. Harris, B. A. Korgel, C. Wang, W. D. Nix, and Y. Cui, Nano Lett. 13, 758 (2013).

${ }^{12}$ L. A. Berla, S. W. Lee, I. Ryu, Y. Cui, and W. D. Nix, J. Power Sources 258, 253 (2014).

${ }^{13}$ M. T. McDowell, S. W. Lee, W. D. Nix, and Y. Cui, Adv. Mater. 25, 4966 (2013).

${ }^{14}$ J. Y. Kwon, J. H. Ryu, and S. M. Oh, Electrochim. Acta 55, 8051 (2010).

${ }^{15}$ L. A. Berla, S. W. Lee, Y. Cui, and W. D. Nix, J. Power Sources 273, 41 (2015).

${ }^{16}$ Q. P. McAllister, K. E. Strawhecker, C. R. Becker, and C. A. Lundgren, J. Power Sources 257, 380 (2014).

${ }^{17}$ A. Kushima, J. Y. Huang, and J. Li, ACS Nano 6, 9425 (2012).

${ }^{18}$ K. Zhao, M. Pharr, S. Cai, J. J. Vlassak, and Z. Suo, J. Am. Ceram. Soc. 94, s226 (2011).

${ }^{19}$ V. A. Sethuraman, M. J. Chon, M. Shimshak, V. Srinivasan, and P. R. Guduru, J. Power Sources 195, 5062 (2010).

${ }^{20}$ S. Huang and T. Zhu, J. Power Sources 196, 3664 (2011).

${ }^{21}$ M. K. Chan, C. Wolverton, and J. P. Greeley, J. Am. Chem. Soc. 134, 14362 (2012).

${ }^{22}$ K. Zhao, W. L. Wang, J. Gregoire, M. Pharr, Z. Suo, J. J. Vlassak, and E. Kaxiras, Nano Lett. 11, 2962 (2011).

${ }^{23}$ R. L. C. Vink, G. T. Barkema, W. F. van der Weg, and N. Mousseau, J. Non-Cryst. Solids 282, 248 (2001).

${ }^{24}$ K. Laaziri, S. Kycia, S. Roorda, M. Chicoine, J. Robertson, J. Wang, and S. Moss, Phys. Rev. Lett. 82, 3460 (1999).

${ }^{25}$ A. France-Lanord, E. Blandre, T. Albaret, S. Merabia, D. Lacroix, and K. Termentzidis, J. Phys.: Condens. Matter 26, 055011 (2014).

${ }^{26}$ E. Holmström, B. Haberl, O. Pakarinen, K. Nordlund, F. Djurabekova, R. Arenal, J. Williams, J. Bradby, T. Petersen, and A. Liu, J. Non-Cryst. Solids 438, 26 (2016).

${ }^{27}$ X. H. Liu, A. V. Davydov, S. X. Mao, S. T. Picraux, S. L. Zhang, J. Li, T. Zhu, J. Y. Huang, J. W. Wang, S. Huang, F. F. Fan, X. Huang, Y. Liu, S. Krylyuk, J. Yoo, and S. A. Dayeh, Nat. Nanotechnol. 7, 749 (2012).

${ }^{28}$ Z. Xie, Z. Ma, Y. Wang, Y. Zhou, and C. Lu, RSC Adv. 6, 22383 (2016).

${ }^{29}$ J. W. Wang, Y. He, F. Fan, X. H. Liu, S. Xia, Y. Liu, C. T. Harris, H. Li, J. Y. Huang, S. X. Mao, and T. Zhu, Nano Lett. 13, 709 (2013).

${ }^{30}$ H. Liao, K. Karki, Y. Zhang, J. Cumings, and Y. Wang, Adv. Mater. 23, 4318 (2011).

${ }^{31}$ H. Ghassemi, M. Au, N. Chen, P. A. Heiden, and R. S. Yassar, ACS Nano 5, 7805 (2011).

${ }^{32}$ G. A. Tritsaris, K. Zhao, O. U. Okeke, and E. Kaxiras, J. Phys. Chem. C 116, 22212 (2012).

${ }^{33}$ F. F. Fan, S. Huang, H. Yang, M. Raju, D. Datta, V. B. Shenoy, A. C. T. van Duin, S. L. Zhang, and T. Zhu, Modell. Simul. Mater. Sci. Eng. 21, 074002 (2013).

${ }^{34}$ Z. Cui, Z. Cui, F. Gao, and J. Qu, J. Power Sources 207, 150 (2012).

${ }^{35}$ V. A. Sethuraman, M. J. Chon, M. Shimshak, N. Van Winkle, and P. R. Guduru, Electrochem. Commun. 12, 1614 (2010).

${ }^{36}$ D. H. Tsai, J. Chem. Phys. 70, 1375 (1979).

${ }^{37}$ M. Zhou, Proc. R. Soc. London, Ser. A 459, 2347 (2003).

${ }^{38}$ I. Ryu, J. W. Choi, Y. Cui, and W. D. Nix, J. Mech. Phys. Solids 59, 1717 (2011).

${ }^{39}$ M. Wang, C. Yan, L. Ma, and N. Hu, Comput. Mater. Sci. 68, 138 (2013). 
${ }^{40}$ M. Wang, C. Yan, L. Ma, N. Hu, and M. Chen, Comput. Mater. Sci. 54, 236 (2012).

${ }^{41}$ S. Plimpton, J. Comput. Phys. 117, 1 (1995).

${ }^{42}$ M. C. Wang, C. Yan, L. Ma, N. Hu, and G. P. Zhang, Comput. Mater. Sci. 75, 69 (2013)

${ }^{43}$ M. C. Wang, C. Yan, D. Galpaya, Z. B. Lai, L. Ma, N. Hu, Q. Yuan, R. X. Bai, and L. M. Zhou, J. Nano Res. 23, 43 (2013).
${ }^{44}$ Y. Xu, M. Wang, N. Hu, J. Bell, and C. Yan, RSC Adv. 6, 28121 (2016).

${ }^{45}$ A. Stukowski, Modell. Simul. Mater. Sci. Eng. 18, 015012 (2010).

${ }^{46}$ W. Humphrey, A. Dalke, and K. Schulten, J. Mol. Graphics 14, 33 (1996).

${ }^{47}$ H. Kim, C.-Y. Chou, J. G. Ekerdt, and G. S. Hwang, J. Phys. Chem. C 115, 2514 (2011).

${ }^{48}$ Y.-H. Yeh, H. Nakashima, H. Kurishita, S. Goto, and H. Yoshinaga, Mater. Trans., JIM 31, 284 (1990). 